$\mathbf{m} / \mathbf{s}$

médecine/sciences $1997 ; 13: 299-300$

\title{
L'ÉTABLISSEMENT FRANÇAIS DES GREFFES
}

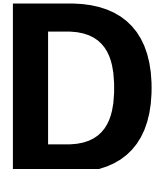

eux ans après sa mise en place, l'Établissement français des Greffes est maintenant en pleine action.

Établissement public d'État fortement décentralisé avec ses sept coordinations inter-régionales, l'Établissement français des Greffes est une agence de régulation, d'encadrement, d'analyse et d'appui d'une activité thérapeutique pratiquée exclusivement dans les Établissements de Santé. La greffe peut se caractériser de manière globale par son efficacité, par un obstacle majeur à sa mise en œuvre, l'obtention du greffon, et par son coût élevé. Plus qu'une simple méthode thérapeutique, elle ouvre également la réflexion sur le corps, la mort, le don, le risque, la justice distributive, la communication, l'éducation et l'avenir de l'homme.

Identifiée comme un domaine de santé publique, la greffe, avec la création de ce nouvel Établissement et selon la volonté du législateur, fait maintenant l'objet de l'attention plus étroite de l'État.

Au-delà du simple passage du monde associatif au giron de l'Etat, l'Établissement français des Greffes témoigne aussi du désir d'unification du champ des greffes et du souhait de mettre en facteur commun ce que partagent les près de 6000 greffes d'organes ou de cellules souches hématopoiétiques et 25000 greffes de tissu faites chaque année en France : les conditions du recueil du consentement à un prélèvement d'éléments du corps humain sur des personnes vivantes ou décédées, le recueil de ces éléments, l'attente des malades, la répartition des greffons, l'objectif de soigner les malades de la meilleure manière possible, avec le moins de risque possible, et pour le moindre coût possible.

L'Établissement français des Greffes a pour interlocuteur principal l'hôpital, universitaire ou non, public ou bien privé. Grâce à son réseau de coordination inter-régionale au contact direct des équipes de prélèvement et de greffes et des coordinateurs hospitaliers, au pôle d'expertise médico-scientifique que constitue l'ensemble du Conseil Médical et Scientifique et du Département Médical et Scientifique, à ses capacités juridiques et en terme de communication et de formation, l'Établissement français des Greffes vise à apporter plusieurs types de services. L'Etablissement participe à l'effort de recherche dans le domaine des greffes et à l'éducation du public et des personnels hospitaliers. Sur le plan opérationnel, il s'agit de la gestion nationale des listes d'attente, de la répartition des greffons et de l'éva- 
luation quantitative, qualitative et économique de ces activités. Sur le plan organisationnel, l'Établissement français des Greffes contribue à la mise en place future de banques de tissus autorisées à l'élaboration de règles de bonnes pratiques de prélèvement, de conservation et de transformation des organes, tissus ou cellules, à la sécurité des greffes et à la coordination au niveau du territoire des activités liées aux greffes.

Nombreux sont aujourd'hui les facteurs de défiance dans l'esprit du public et peut-être aussi dans celui de certains lecteurs de médecine/sciences: qu'est-ce que la mort encéphalique ? Quel respect pour le corps humain lors du prélèvement d'organes ou de tissus ? Quelle justice dans la répartition des greffons? Quels sont les résultats de ces greffes dont le coût est élevé et dont la pratique s'appuie sur un assentiment souvent extraordinaire dans le contexte d'une mort brutale ? En 1996, une gestion plus rigoureuse de la liste d'attente des patients devant subir une greffe d'organes a été mise en place. L'attention des équipes a été attirée, dans un esprit de santé publique et compte tenu du manque actuel de greffons, sur la nécessaire maîtrise de l'accueil des malades non résidents candidats à la greffe. Les règles de répartition ont été homologuées par le ministre chargé de la Santé. Un état des lieux du secteur des greffes de tissus, de moelle osseuse, de cellules a été effectué

Pour 1997, les objectifs principaux sont les suivants : accroître le recueil des organes et tissus en vue de greffe grâce au renforcement des coordinations hospitalières gérant les prélèvements et à l'appui des anesthésistes et réanimateurs; poursuivre la construction du secteur des tissus et des cellules, en mettant en place la liste nationale d'attente pour la greffe de cornée ; enregistrer tous les malades devant subir une greffe de cellules souches hématopoïétiques et enfin, définir une règle du jeu pour l'évaluation des résultats des greffes qui obtienne l'approbation de toutes les équipes hospitalières quant à ses critères, ses modalités et ses objectifs. Il faudra aussi poursuivre la mobilisation autour de la question des greffes de tous les acteurs concernés, non seulement, évidemment, des équipes hospitalières, mais aussi de tous ceux qui contribuent à l'état d'esprit d'un hôpital face à la greffe, médecins, pharmaciens, services administratifs, techniques ou logistiques.

Enfin l'Établissement se fixe pour but d'informer précisement le public sur la mise en place du registre des refus, prévu par la loi, et de participer à l'éducation des jeunes sur ces questions. Cela permettra peut-être de surmonter un à un les facteurs expliquant la défiance actuelle du public, dans le but d'offrir toutes leurs chances à un plus grand nombre de malades

\section{INSTITUT COCHIN DE GÉNÉTIQUE MOLÉCULAIRE}

\section{JOURNÉE JEAN-CLAUDE DREYFUS DE GÉNÉTIQUE ET DE PATHOLOGIE MOLÉCULAIRES RÉCEPTEURS et MALADIES \\ RECEPTORS and DISEASES \\ Vendredi 19 septembre 1997}

Grand Amphithéâtre de la Faculté de Médecine Cochin Port-Royal

24, rue du Faubourg-St-Jacques - 75014 PARIS, France

$$
\text { Session } \mathrm{n}^{\circ} 1 \text { : }
$$

Récepteurs et Maladies du Métabolisme

Receptors and metabolic diseases

Président : Donny A. STROSBERG, UPR 0415 Cnrs, ICGM, PARIS

Session $n^{\circ} 2$ :

Récepteurs et Maladies Neuropsychiatriques

Receptors and neuropsychiatric diseases

Président : Bruno GIROS, U. 288 Inserm, CHU PITIÉ-SALPÊTRIÈRE, PARIS

Session $n^{\circ} 3$ :

Récepteurs et Maladies Microbiennes et Parasitaires

Receptors and microbial or parasitic diseases

Président : Philippe SANSONETTI, U. 389 Inserm, INSTITUT PASTEUR, PARIS

$$
\text { Session } \mathrm{n}^{\circ} 4 \text { : }
$$

Récepteurs et Maladies Virales

Receptors and viral diseases

Président : Gilbert VASSART, ULB, CAMPUS HOSPITAL ERASME, BRUXELLES

Renseignements et Inscriptions : APEMM CONGRÈS (Axel KAHN) 